\title{
A Soft Computing Model for Predicting Students' Academic Performance in Tertiary Institutions
}

\author{
Olutayo Boyinbode \\ Department of Information \\ Technology, Federal University of \\ Technology, Akure, Nigeria
}

\author{
Oluwaseun Ayankunle \\ Department of Computer Science, \\ Federal University of Technology, \\ Akure, Nigeria
}

\author{
Olumide Obe \\ Department of Computer Science, \\ Federal University of Technology, \\ Akure, Nigeria
}

\begin{abstract}
Educational Institutions are striving to foster the prediction of student performance into their educational sector for better students' support, this is achieved by discovering students with lower performance and making additional efforts to improve their performance. Assessing and predicting students' performance enhance academic performance and is a catalyst to delivering high quality education. Soft computing is a promising technique used in solving prediction problems to enhance academic performance in educational sectors. This paper implemented a soft computing model (Adaptive Neuro Fuzzy Model using Levenberg-Marquardt algorithm) for predicting Students' Academic Performance in Tertiary Institutions. The system was implemented using MATLAB 2017a. The developed model has an accuracy of 99.33\%, which is the highest, when compared with some previous works.
\end{abstract}

\section{General Terms}

Soft Computing, Neural networks, Fuzzy Logic, Decision Tree Algorithm

\section{Keywords}

Adaptive Neuro-Fuzzy Inference System, LevenbergMarquardt Algorithms, Student Performance, Assimilation Rate (AR), Cramming ability (CA), Hours Student Read Per day (HSRPD), Solving Past Questions Frequently (SPQF), Class Attendance Rate (CAR), Financial Strength (FS).

\section{INTRODUCTION}

Students' performance prediction has evolved as a critical factor in attaining high quality education. Educational Institutions recognize the importance of student performance and are greatly motivated to predict their student performance, so as to be able to offer good support for their students; this is achieved by locating students with lower performance and rendering necessary assistance to improve their performance. Prediction simply means forming an opinion, about what will happen in the future. One of the most potent ways of solving students' performance prediction problems is through the application of Soft Computing (SC) techniques. SC is an approach to computing, which allows the human brain to learn and reason in an environment of uncertainty and imprecision [1]. Soft Computing techniques have their roots in Artificial Intelligent (AI), Fuzzy Logic (FL) and Neural Network (NN). Many SC methods that were applied in the past, had one some short coming or the other, therefore, this work embraced Adaptive Neuro Fuzzy Model using Levenberg -Marquardt algorithm to obtain an effective students performance prediction model [2]. An Adaptive Neuro Fuzzy System (ANFIS) has rapid learning rate, system performance overhead is flexible, and also the membership functions and training epochs can be changed [3]. ANFIS has achieved great success, due to the following: It contains fuzzy IF-THEN rules to explore the behaviour of different systems. It also excludes the inclusion of human expertise. There are several methods used in the learning algorithms: Gradient Decent Backpropagation, Radial Basis Function, and the fastest known technique called Levenberg-Marquardt (LM) [4]. LM algorithm is a promising method to train Fuzzy Neural Network (FNNs) and it has been shown, that it exhibits a very good performance and better forecasting capability [5]. This research implements an Adaptive Neuro-Fuzzy Inference System (ANFIS) model for predicting students' academic performance in tertiary institutions. using student's cognitive attributes (i.e. assimilation ability etc.), personal factors (Financial strength and actions, Class Attendance, Rate of Solving Past Questions). This model aimed at addressing some current key problems in the educational sector

\section{RELATED WORKS}

Pandey and Sharma [8] developed a Decision Tree Algorithm (DTA) to predict the future grades of the students in their final examinations, the data mining approach has the following shortcomings: A little change in the data can cause an enormous change in the structure of the decision tree, hereby affecting the stability. Sometimes calculation can be more complicated when compared to other algorithms. The training time for the model is higher and cost intensive. Márquez-Vera et al. [6] Predicted student failure at school, using genetic programming (GP) and different data mining methods with several dimension of data. Ajayi and Akindele [9] developed a Neuro-Fuzzy Model for Predicting Students Performance in Object-Oriented Programming Courses. Even though the study predicted students' academic performance in ObjectOriented Programming Courses, using a Neuro-Fuzzy Model, the dataset (92) used in the study was very small to guarantee accuracy of the prediction and the study was not evaluated. Oluyege [10] developed a Neuro-Fuzzy Model for Predicting Students' Academic Performance in Nigerian Tertiary Institutions, although the study proved that neuro-fuzzy system can improve the output of students' performance prediction, however the developed model has high computation time. Arora and Saini [7] developed a Probabilistic Neural Network hybridized with fuzzy logic to predict students' performance and to sort them according to their striking features. The work was motivated by the need to sort and forecast students' academic performance using arithmetical and statistical techniques that may proffers the best way to assess human accession of knowledge and prowess. The developed system suffers a low classification accuracy. This research work is therefore motivated by the need to proffer solutions to the limitations encountered in the previous works. 


\section{RESEARCH METHODOLOGY}

Data were collected from online questionnaire, with the help of google form. The data include various factors that may affect student academic performance, such as Cramming Ability (CA), Study intensively over a short period of time just before an examination, Assimilation Rate (AR) (the process of taking in and fully understanding information or ideas), Do you do Assignment regularly?, What is your Financial Strength?, Hours Student Read Per day (HSRPD), How often do you attend Class in rating of 1-5?, How often do you solve past question?, When is your most comfortable reading period, Where did you grow up?, What is your eating/nutrition pattern $(1: 1: 1$ implies breakfast: lunch: dinner)?, How often do you take Alcohol?, How often do you use Drugs like caffeine/ cocaine/codeine?, Do your family have history of mental illness/sickness?, What was your average performance in secondary school?

\subsection{Data Preprocessing}

Data Preprocessing: The acquired dataset was preprocessed using Microsoft Excel and MATLAB Data Tool. The dataset values for the input variables were converted from string variables to respective integer codes so as to make it usable with the ANFIS algorithm. The developed system has multi inputs and single output. The dataset codes used are as below; Inputs variables, their representation and integer values

- Solving Past Questions Frequently (SPQF): Rarely - 1, Averagely - 2, Very Often - 3

- Hours Student Read Per day (HSRPD): one hour - 1, two hours - 2, three hours - 3, (four-six) hours - 4

- Cramming Ability (CA): low - one (1), average - two (2), good - three (3), very good - four (4)

- Assimilation Rate (AR): average - two (2), good - three (3), very good - four (4)

- Financial Strength (FS): very weak - one (1), weak Two (2), average - three (3), strong - four (4), very strong-five (5)
- Average Performance in Secondary School (APSS): 50 $60 \%-1,60-70 \%-2,80-100 \%-3$

- Assignment Frequently (AF): Averagely -1, Very Often2

- Class Attendance Rate (CAR): 1-5 Cumulative Grade Point Average (CGPA) of students as the Output.

\subsection{Data Training and Testing}

The dataset was divided into two parts which are the training (80\%) and testing (20\%). Total number of datasets used is 325. The training and testing part of the dataset was preprocessed by the process of normalization and its represented in equation $\{1\}$.

$X_{l}=\frac{X_{l}-\mu}{\sigma}\{1\}$

where $X_{l}$ is the normalized sample, $\mathrm{x}_{l}$ is the input data, $\sigma$ is the standard deviation of dataset and $\mu$ is the sample mean. After this, the model was trained via Levenberg-Marquardt algorithm, as represented in equation $\{2\}$. The data was subjected to data mining using Neuro fuzzy technique implemented as a Sugeno type ANFIS system.

$J=\frac{\partial_{e i}}{\partial_{w j}}$ for $\mathrm{i}=1 \ldots, \mathrm{m}$ and $\mathrm{j}=\mathrm{i} \ldots . ., \mathrm{n}$

where $m$ is the number of instances in the dataset, $n$ is the numbers of parameter in the neural network and, $J$ is the Jacobian matrix. At the end, the accuracy of the model was validated and the system was implemented using MATLAB programming language.

\section{RESULTS AND ANALYSIS}

The system interface for ANFIS model is shown in Figure 1. Table 1 shows some of the actual and the predicted data result obtained; after training, testing and prediction. Its graph is shown in Figure 2.

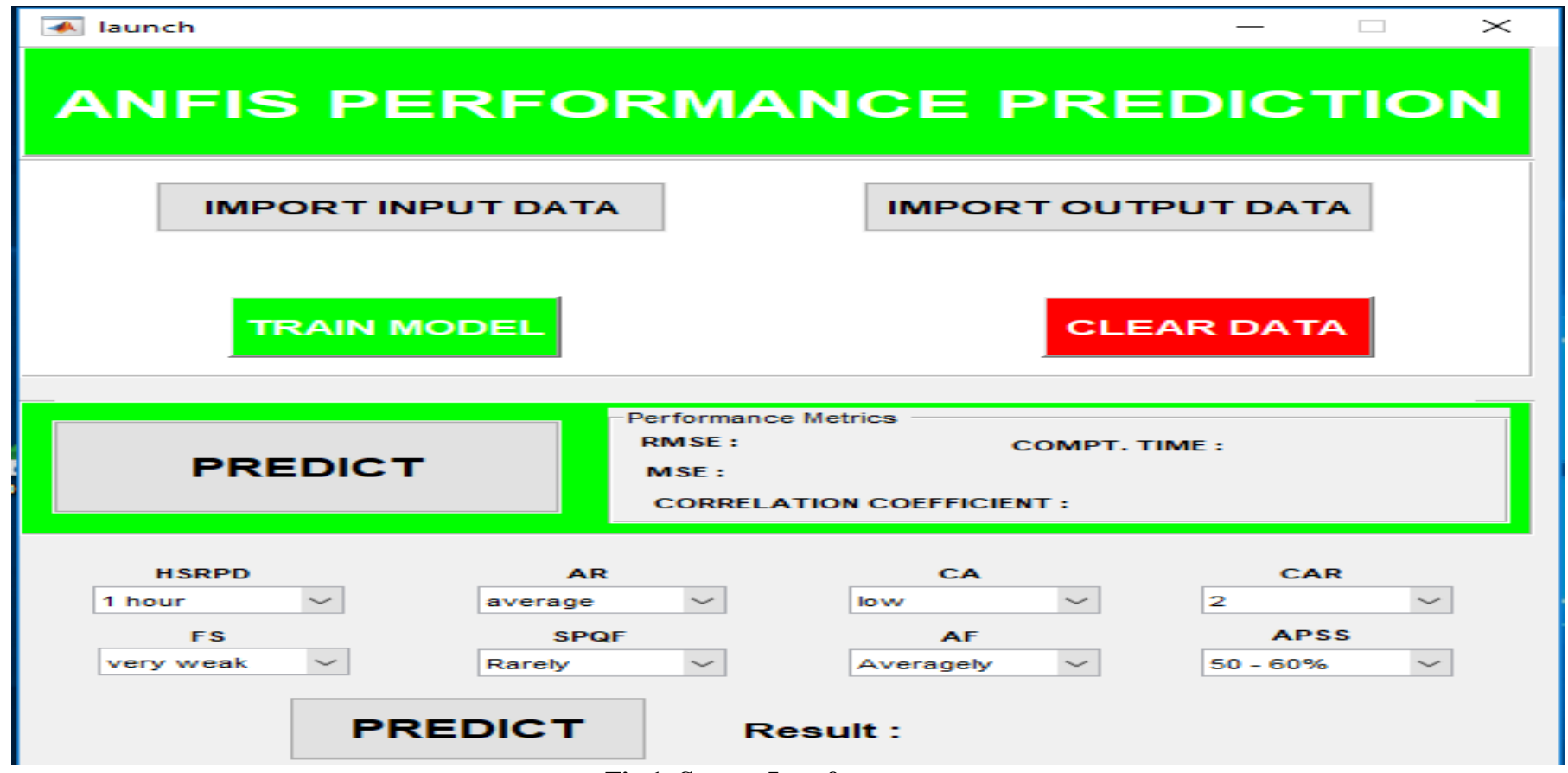

Fig 1: System Interface 
Table 1. Results of Some Actual and Predicted data

\begin{tabular}{|c|c|c|}
\hline $\mathrm{S} / \mathrm{N}$ & Actual Data & Predicted Data \\
\hline 1 & 4.2 & 4.191599612 \\
\hline 2 & 3.13 & 3.123739903 \\
\hline 3 & 3.19 & 3.183619945 \\
\hline 4 & 2.8 & 2.794400016 \\
\hline 5 & 3.9 & 3.89219797 \\
\hline 6 & 3 & 2.993999663 \\
\hline 7 & 4.27 & 4.261459687 \\
\hline 8 & 3.5 & 3.492998373 \\
\hline 9 & 3.5 & 3.492998373 \\
\hline 10 & 2.79 & 2.784419812 \\
\hline 11 & 2.79 & 2.784421422 \\
\hline 12 & 3.55 & 3.54289987 \\
\hline 13 & 3.9 & 3.89219797 \\
\hline 14 & 3 & 2.993999663 \\
\hline 15 & 4.27 & 4.261459687 \\
\hline 16 & 3.5 & 3.492998373 \\
\hline 17 & 3.5 & 3.492998373 \\
\hline 18 & 2.79 & 2.784419812 \\
\hline 19 & 2.79 & 2.784421422 \\
\hline 20 & 3.55 & 3.54289987 \\
\hline 21 & 2.7 & 2.694599933 \\
\hline 22 & 4.2 & 4.191599612 \\
\hline 23 & 3.13 & 3.123739903 \\
\hline 24 & 3.19 & 3.183619945 \\
\hline 25 & 2.8 & 2.794400016 \\
\hline 26 & 3.9 & 3.89219797 \\
\hline 27 & 3 & 2.993999663 \\
\hline 28 & 2.7 & 2.694599933 \\
\hline 29 & 4.2 & 4.191599612 \\
\hline 30 & 3.13 & 3.123739903 \\
\hline 31 & 3.19 & 3.183619945 \\
\hline 32 & 2.8 & 2.794400016 \\
\hline 33 & 3.9 & 3.89219797 \\
\hline 34 & 3 & 2.993999663 \\
\hline 35 & 4.27 & 4.261459687 \\
\hline 36 & 3.5 & 3.492998373 \\
\hline 37 & 3.5 & 3.492998373 \\
\hline 38 & 2.79 & 2.784419812 \\
\hline 39 & 2.79 & 2.784421422 \\
\hline 40 & 3.55 & 3.54289987 \\
\hline
\end{tabular}




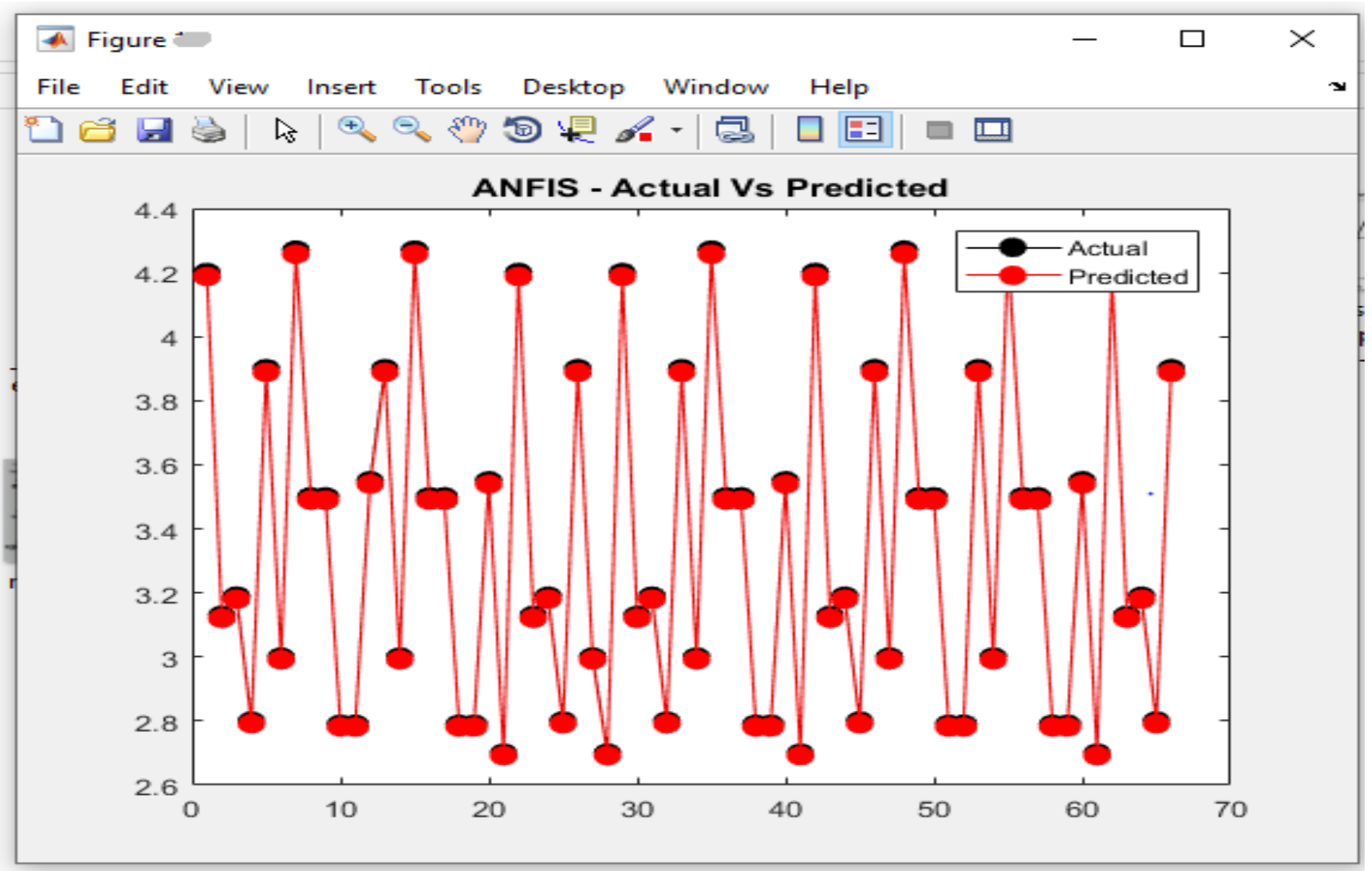

Fig 2: Predicted and Actual Result Graph

The ANFIS Model with 3 layers; layer 1 is the input layer with 8 inputs, layer 2 is the hidden neuron with 256 rules, while layer 3 is the output layer which is the output of the model (see Figure 3). The eight input variables used for the study are Assimilation Rate (AR), Cramming ability (CA),
Hours Student Read Per day (HSRPD), Solving Past Questions Frequently (SPQF), Class Attendance Rate (CAR), Financial Strength (FS), Assignment Frequently (AF), Average Performance in Secondary School - (APSS) and CGPA for the output (see figure 4).

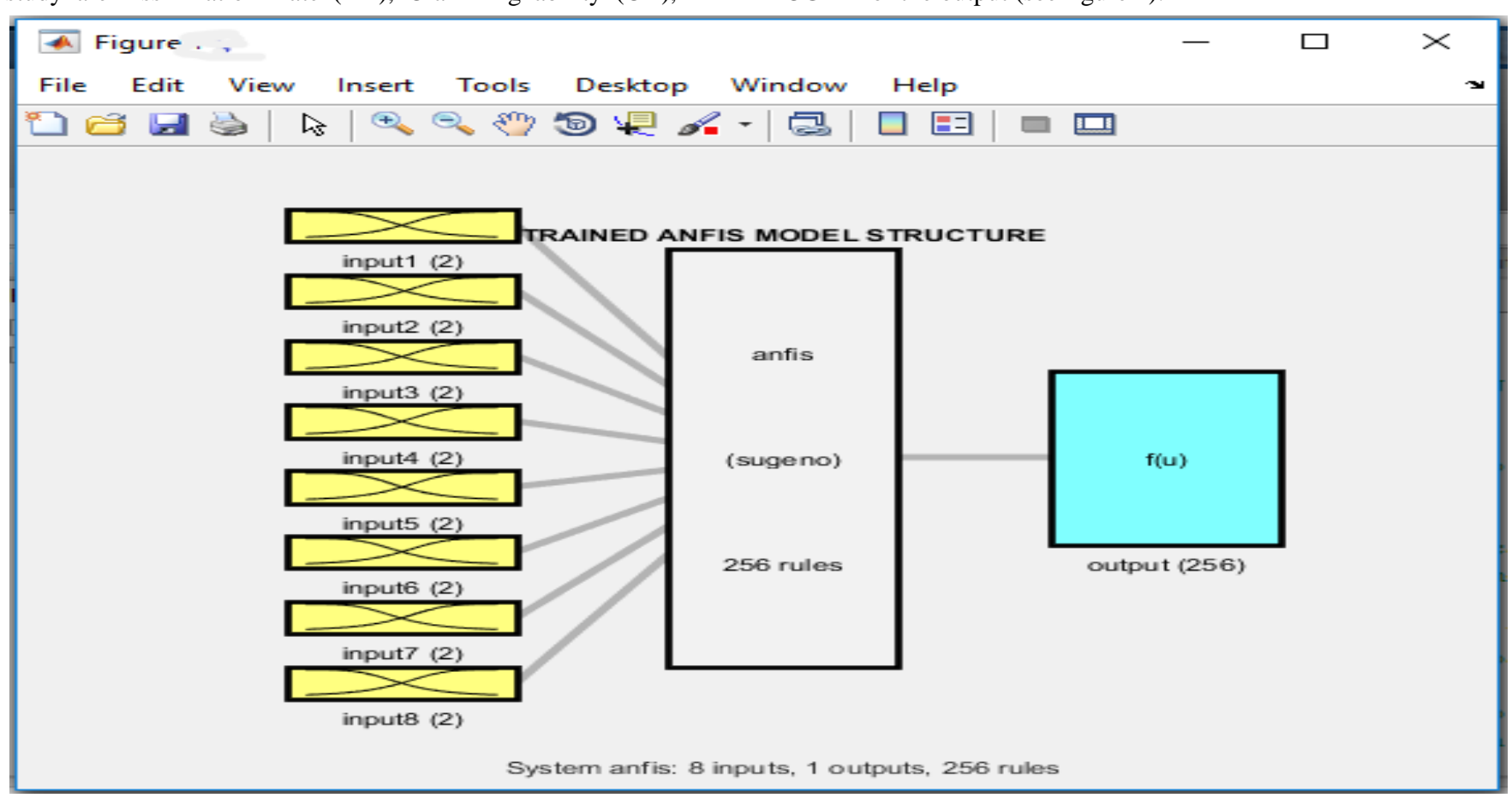

Fig 3: ANFIS Model 


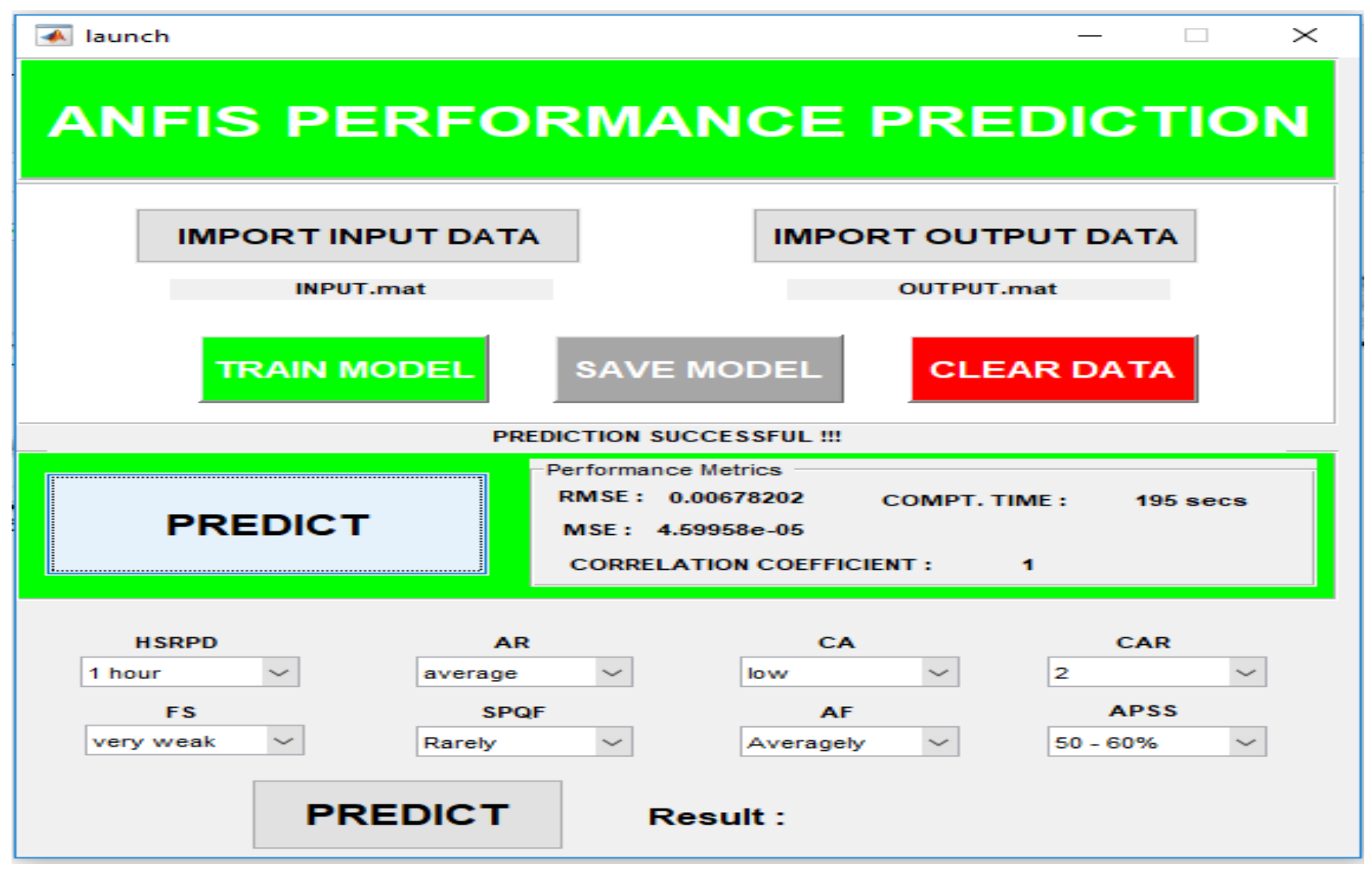

Fig 4: Prediction Result Interface

\section{PERFORMANCE EVALUATION}

The prediction time is the time taken for the proposed system to predict the accuracy of the student performance. It is measured in seconds. This is illustrated by comparing the prediction time of the developed model with Oluyege (2019) and Arora \& Saini (2013) models (see table 2 and figure 5).

Table 2. Comparism of Computation Time (s)

\begin{tabular}{|c|c|}
\hline PROJECT WORK & TIME (seconds) \\
\hline Developed work & 195 \\
\hline Oluyege (2019) & 3588 \\
\hline Arora \& Saini (2013) & 5400 \\
\hline
\end{tabular}

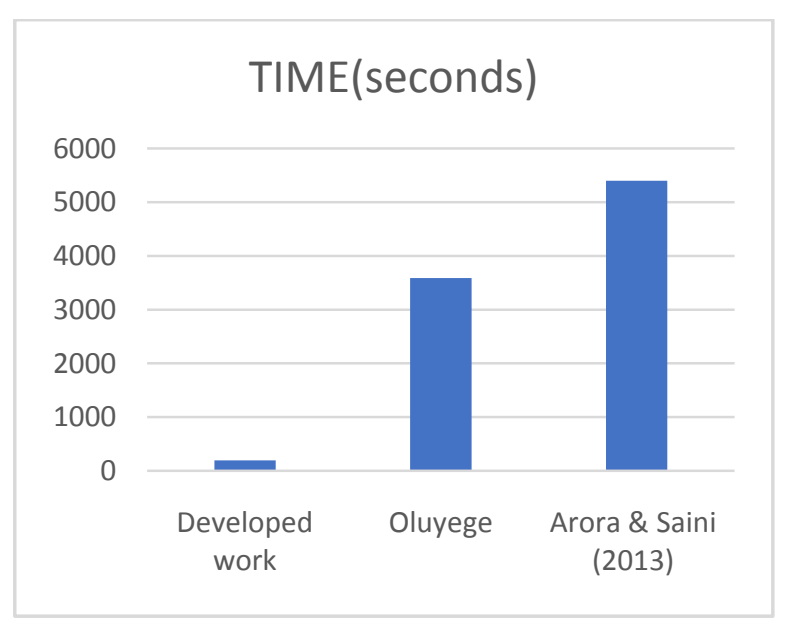

Fig 5: Comparism of Computation Time(s)
The Root Mean Square Error (RMSE) is used to estimate the precision of the students' performance prediction by computing the variance between performance of the actual data values and the predicted ones by the model. It is depicted as follows:

$R M S E=\sqrt{\frac{\sum_{l=1}^{n}\left(X_{\text {obs },}-X_{\text {pred }, l}\right)^{2}}{n}}$

where $X_{o b s, l}$ is the observed performance and $X_{\text {pred }, l}$ is the predicted performance. Lower values of RMSE indicate better fit. The developed model has an RMSE of 0.00678202(s) while previous works had the following RMSE: Oluyege 2019; 0.1189(s), Chen et al., 2014; 0.4819(s), Do and Chen (2013); 0.5157(s) (see table 3 and figure 6). The developed model shows that ANFIS trained with LM algorithm exhibits faster training speed and better estimation ability.

Table 3. Comparism of Prediction RMSE(s)

\begin{tabular}{|c|c|}
\hline Project Work & RMSE \\
\hline Developed Work & 0.0068 \\
\hline Oluyege (2019) & 0.1126 \\
\hline Chen et al 2014 & 0.4819 \\
\hline Do \& Chen (2013). & 0.5157 \\
\hline
\end{tabular}




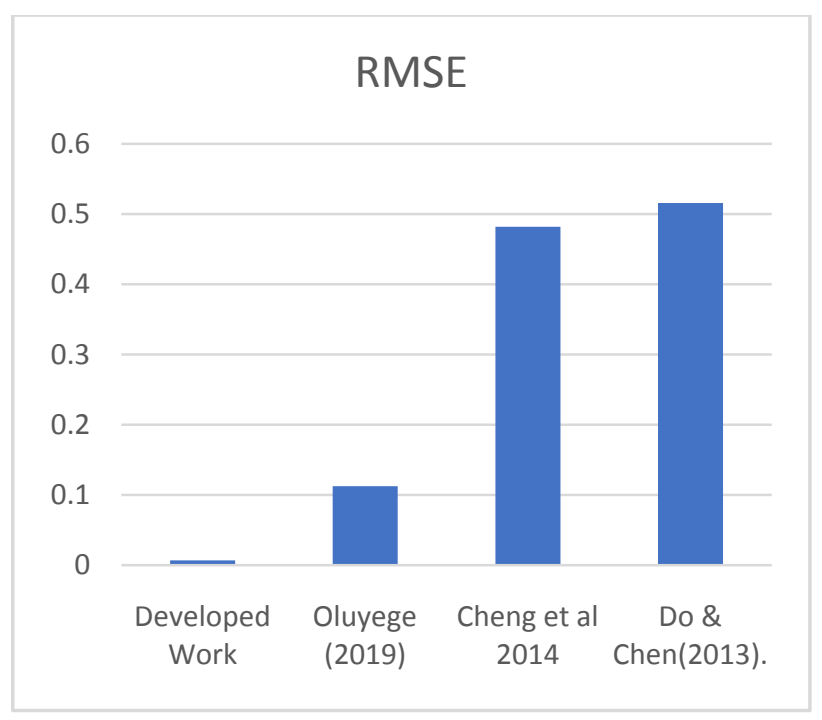

Fig 6: Comparism of Computation RMSE(s) Graph

\section{CONCLUSION}

This research work established an Adaptive Neuro-Fuzzy Inference System (ANFIS) model for effective prediction of student performance using Levenberg Marquardt algorithm. The developed model shows that FNNs trained with LM algorithm exhibits rapid training speed and more efficient estimation capability. The system was implemented using MATLAB 2017a and Excel 2016 on Windows 10 Operating System platform. The computation time for the developed model was 195 seconds, which is 94\% more efficient compared to the previous work. The developed model also had an RMSE of 0.00678202 (s) which is $94.3 \%$ better, when compared to the previous work.

\section{REFERENCES}

[1] Panda M. and Patra M.R. 2013 Soft Computing: Concepts and Techniques https://www.researchgate.net/publication/280083168

[2] Do, Q. H., \& Chen, J. F. (2013). A neuro-fuzzy approach in the classification of students' academic performance. Computational intelligence and neuroscience, 2013, 6 .

[3] Satwanti Devi, Sanjay Kumar ad Govind Singh Kushwaha (2016) An Adaptive Neuro Fuzzy Inference System for Prediction of Anxiety of Students.
International Conference on Advanced Computational Intelligence (ICACI 2016) 7-13

[4] Arsad, P. M., \& Buniyamin, N. (2013, November). A neural network students' performance prediction model (NNSPPM). In 2013 IEEE International Conference on Smart Instrumentation, Measurement and Applications (ICSIMA) (pp. 1-5). IEEE.

[5] Khanesar, M. A., Kayacan, E., Teshnehlab, M., \& Kaynak, O. (2011, April). Levenberg Marquardt algorithm for the training of type-2 fuzzy neuro systems with a novel type-2 fuzzy membership function. In 2011 IEEE symposium on advances in type-2 fuzzy logic systems (T2FUZZ) (pp. 88-93). IEEE.

[6] Márquez-Vera, C., Cano, A., Romero, C., and Ventura, S. (2013). Predicting student failure at school using genetic programming and different data mining approaches with high dimensional and imbalanced data. Applied intelligence, 38(3), 315-330.

[7] Arora, N., \& Saini, J. R. (2013). A fuzzy probabilistic neural network for student's academic performance prediction. International Journal of Innovative Research in Science, Engineering and Technology, 2(9), 44254432.

[8] Pandey, M., \& Sharma, V. K. (2013). A decision tree algorithm pertaining to the student performance analysis and prediction. International Journal of Computer Applications, 61(13).

[9] Olusola Olajide Ajayi and Temitope Clement Akindele. A Neuro-Fuzzy Model for Predicting Students Performance in Object-Oriented Programming Courses. International Journal of Applied Information Systems 12(21):26-32, June 2019.

[10] Oluyege (2019) A Neuro-Fuzzy Model for Predicting Students' Academic Performance in Nigerian Tertiary Institutions" Master of Technology Computer Science:Thesis Department of Computer Science, School of Computing, The Federal University of Technology, Akure, Nigeria.

[11] Chen, J. F., Hsieh, H. N., \& Do, Q. (2014). Predicting student academic performance: A comparison of two meta-heuristic algorithms inspired by cuckoo birds for training neural networks. Algorithms, 7(4), 538-553. 